\title{
A SIMPLE METHOD FOR THE GENERATION OF ORGAN AND VESSEL CONTOURS FROM ROENTGENOGRAPHIC OR FLUOROSCOPIC IMAGES*
}

\author{
John D. Newell, Robert A. Keller, and Norman A. Baily \\ University of California, San Diego \\ Department of Radiology, La Jolla, California 92037
}

\begin{abstract}
A simple method for outlining or contouring any area defined by a change in film density or fluoroscopic screen intensity is described. The entire process, except for the positioning of an electronic window, is accomplished using a small computer having appropriate software. The electronic window is operator positioned over the area to be processed. The only requirement is that the window be large enough to encompass total area to be considered.

\section{INTRODUCTION}

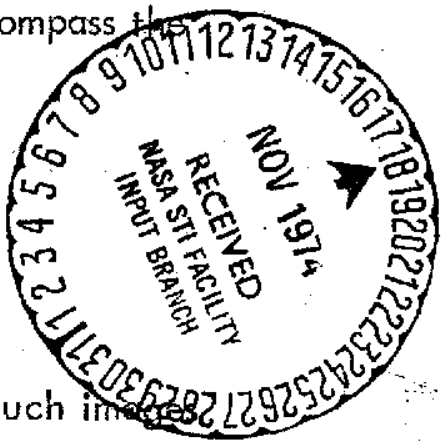

Both in single radiological images and in time sequences of such in 28292 a great deal of quantitative data is incorporated as changes of density or intensity. These changes are indicative of physiological change and/or function, and are derivable from the size and/or shape of these structures.
\end{abstract}

* Work partially supported by NASA Grant No. NGR 05-009-257. 
Dynamic information is obtainable from time sequences of such images. A first step in extracting such information in most cases is to provide the computer with information concerning the boundaries. In the case of single images, both areas and volumes can be obtained. In the case of time sequences of such images changes in these quantities as a function of time can be computed and plotted automatically, velocity and acceleration of the boundaries are readily obtained from such sequences and analysis of the frequency components of such motions can also be readily computed. In all cases, the accuracy, and indeed the availability of such data depends on the ability to extract contours with good accuracy. Trenholm, ef $\mathrm{al}^{1}{ }^{1}$ have shown that when a contour has been defined and the gray scale level of each point within the contour is known, accurate values for volumes can also be easily obtained. Using digitized values and some combination of both temporal and spatial averaging to increase the signal-to-noise ratio, even higher accuracy should result since small gray scale differences not detectable by analog techniques will now be meaningful. 2 This ability combined with an accurate contour defining the outline in a single plane could produce highly accurate volumetric data.

In general, the method to be described works best where high contrasts exist. However, since even a minimal amount of statistical averaging provides a value having a standard deviation in the determination of a mean gray level of \pm 1 percent or approximately \pm 0.3 gray levels, we feel that the method should certainly be useful for structures outlined by changes of gray level corresponding to about twice this value. 2 


\section{METHOD}

The basic method used in the work to be presented in this paper is based on two techniques presented in the book by Rosenfeld. ${ }^{3}$ These are a calculation of the gradient of each point in our digitized image and a thresholding technique. This is then followed by a set of algorithms designed to thin out the number of elements defining the contours.

The first step in the process is the digitization of the image or section of image to be contoured. In other words we produce a picture function $f(x, y)$ having a range of values from $0-31$. These 32 gray levels correspond to the range of fluoroscopic brightriess or roentgenographic densities found in the image to be processed. Various techniques for eliminating statistically independent electronic noise such as spatial averaging, ${ }^{2}$ or multiple digitization can be employed to yield values for $f(x, y)$ having a high degree of reliability especially with respect to their relative values. The second step involves a numerical calculation to obtain a modified gradient of $f(x, y)$ for all values of $x$ and $y$. The directional derivative of each point was calculated using the eight closest neighbors to the point of calculation. For example, given an array of nine picture elements arranged in the following manner:

$$
\begin{aligned}
& A_{x-1, y+1} A_{x, y+1} A_{x+1, y+1} \\
& A_{x-1, y} A_{x, y} A_{x+1, y} \\
& A_{x-1, y-1} A_{x, y-1} A_{x+1, y-1}
\end{aligned}
$$

the gradient of $A_{x, y}$ was computed in the following manner, 3 


$$
\begin{aligned}
& \| \overrightarrow{\operatorname{GRAD}}\left(A_{x, y}\right) \mid=1 / 3\left[\mid\left(A_{x-1, y+1}+A_{x, y+1}+A_{x+1, y+1}\right)-\right. \\
& \left(A_{x-1, y-1}+A_{x, y-1}+A_{x+1, y-1}\right)|+|\left(A_{x-1, y+1}+A_{x-1, y}+\right. \\
& \left.\left.A_{x-1, y-1}\right)-\left(A_{x+1, y+1}+A_{x+1, y}+A_{x+1, y-1}\right) \mid\right]
\end{aligned}
$$

To obtain a contour this matrix of values of the gradient of the original picture function is examined for a selected minimum value. This operation is identical to that usually called thresholding which is employed to make features of greater brightness stand out or even to outline areas. In our case the threshold is applied to the values of the gradient rather than the values of $f(x, y)$. In the examples to be illustrated the threshold value for the gradient which we applied was equivalent to four gradient levels. That is, if the gradient of a picture element did not exceed 10 percent of the gray scale range its value was set equal to zero. This procedure produces confours which in general are thicker than one would desire. To improve the resolution one must narrow down the number of picture elements still retained in the gradient matrix.

In order to eliminate noise spikes, artifacts, etc. from the gradient matrix of the picture elements, the following thinning algorithm was used. Each value of the gradient function $g(x, y)$ is treated as the center point of a $3 \times 3$ array. Each array is examined and each value of $g(x, y)$ that has less than two non-zero values in the $3 \times 3$ array is set equal to zero at the end of this first step of the total procedure. Thus isolated and peripheral elements are eliminated. This is then followed by zeroing all points which have four or more nearest neighbors who themselves have more non-zero neighbors than the point being examined. This algorithm is applied twice and then followed by a gradient maximum 
following algorithm designed to eliminate all but the greatesf values of the gradients remaining in the matrix.

The gradient maximum following algorithm operates in the following way. Each remaining point in the $g(x, y)$ matrix of the gradient of the picture elements is considered as the center of a $3 \times 3$ array of its nearest neighbors as done earlier. The maximum value of the nine gradients is compared to the value of the gradient under consideration and if the maximum value exceeds the gradient by three units then the point is zeroed. The derived contour then is just the points remaining with non-zero values in the matrix representing $g(x, y)$.

\section{RESULTS}

The contouring method described above has been fested on a number of different anatomical structures such as the heart, kidney, brain, and gallbladder. Two of these will be illustrated below.

Fig. I is an example of a cardiovascular application. Since no appropriate roentgenogram was available for our use, recourse was made to the published literature. $4 \mathrm{~A}$, is a selective left ventricular angiogram, lateral projection, and shows a ventricular septal defect. To obtain this picture a negative of the illustration given in reference 4 was used. $B$, is the computer -generated contour. It is important to note that the program has even delineated contours of relatively low contrast as well as those of relatively high contrast.

Fig. 2 is an example of the method's use in a genito-urinary application. A, is a print made from the original roentgenogram obtained during selective arteriography of the left kidney, and $B$, is the resultant computer-generated contour. 


\section{DISCUSSION}

The simple method presented for extracting contours representing density changes of roentgenograms or fluoroscopic brightness changes should be useful for extracting physiological data in the form of organ areas and volumes. The digital data residing in the computer is easily utilized for computations of enclosed areas or the calculation of dynamic changes. Extraneous areas such as those produced by catheters, bony structures, etc. can be eliminated by operator intervention before beginning the computation of such areas. Since gray scale information on each of the picture elements enclosed is also stored in the computer, volumes of such structures. can be calculated using the method of Trenholm, et al. ${ }^{1}$

The method is superior to simple thresholding of gray levels as described by Robb ${ }^{5}$ in that the use of a gradient function allows one to operate with lower contrast levels. It is also faster and less tedious than the method used by Hall, et al, ${ }^{6}$ which again basically uses simple thresholding techniques.

A more recent publication by Clayton, ef al, ${ }^{7}$ details a method using a one-dimensional gradient and a matched gradient filter. This method was designed for use with a large computer in contrast to our method which was designed for use with a small computer in a clinical environment. While their program can probably be made to operate using a small computer, the total operation would be quite slow. Also, the use of a matched gradient filter restricts its use to a specific application depending on specificity of the filter. For highly specific filters, new matches would have to be inserted for most changes in anatomical site application.

The accuracy and applicability of each program deserves further investigation. 


\section{CONCLUSIONS}

1. A simple, and relatively fast method for generating contours from either roentgenograms or video disc recorded fluoroscopic images has been shown to be satisfactory for delineating differences in density or brightness as small as two gray levels (on a scale of $0-31$ ).

2. The computer capacity required is low so that the method is capable of implementation in a clinical environment.

3. Further development of the program with improvement of the algorithms used should produce sharper delineation of the boundaries. 


\section{REFERENCES}

1. B.G. Trenholm, D.A. Winter, G.D. Reimer, D. Mymin, E.L. Lansdown, and G.P. Sharma, Radiology 112, 299 (1974).

2. R.L. Crepeau and N.A. Baily, Proc. of the 12th San Diego Biomed. Symp., 277 (1973).

3. A. Rosenfeld, Picture Processing by Computer (Academic Press, New York, 1969).

4. M.G. Baron, Radiol. Clinics of No. America 6, 353 (1968).

5. R.A. Robb, Proc. of the San Diego Biomed. Symp. 10, 235 (1971).

6. D.L. Hall, G.S. Lodwick, R.P. Kruger, S.J. Dwyer, and J.R. Townes, Radiology 101, 491 (1971).

7. P.D. Clayton, L.D. Harris, S.R. Rumel, and H.R. Warner, Computers and Biomed. Res. 7, 369 (1974). 


\section{FIGURE.CAPTIONS}

Fig. 1: A, selective left ventricular angiogram, lateral projection showing septal defect. B, computer generated contour.

Fig. 2: A, roentgenogram of a selective arteriogram of the left kidney. B, computer-generated contour. 


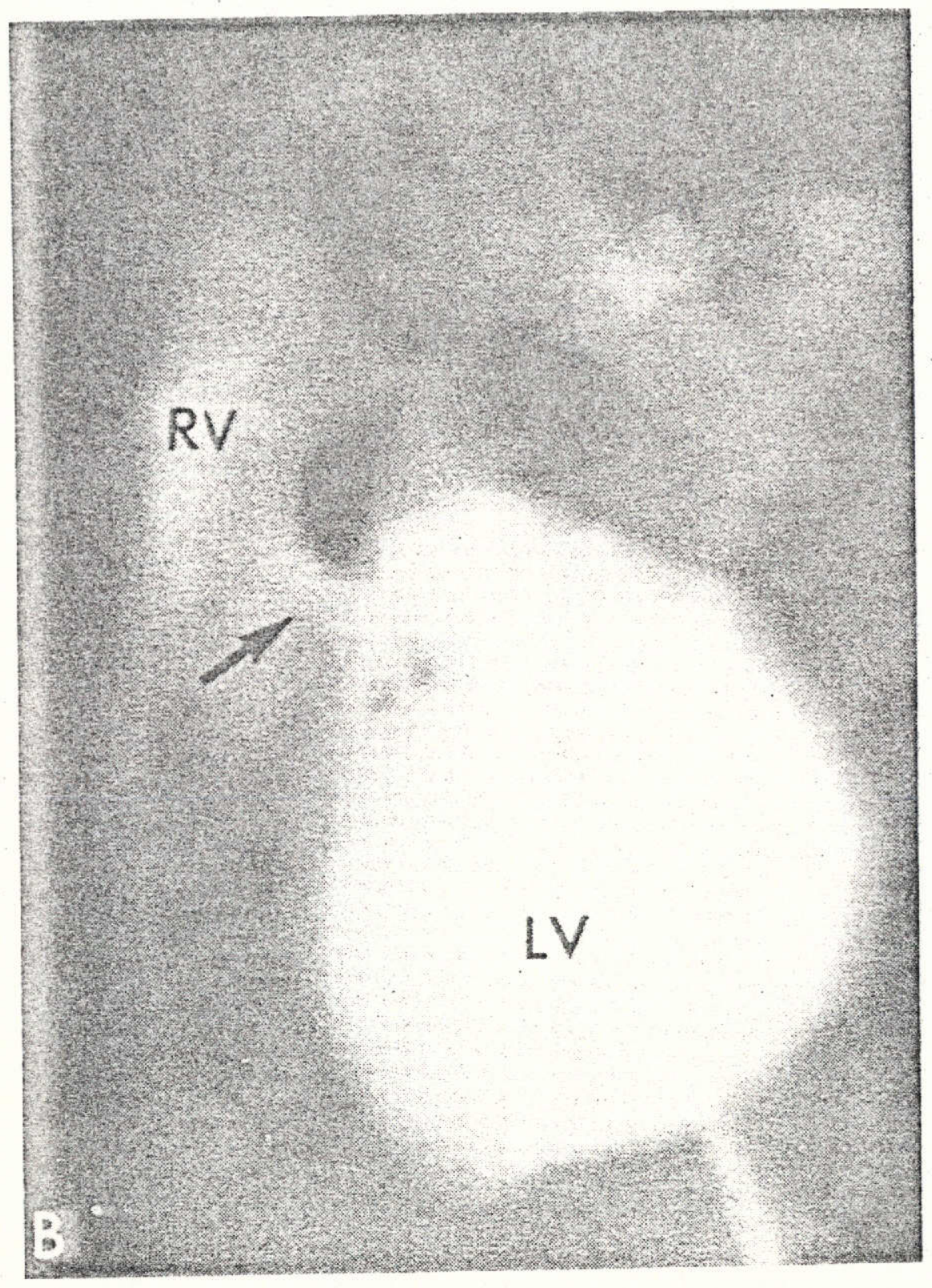

Fig. IA

\section{REPRODUCIBILITY OF THE ORIGINAL PAGE IS POOR}




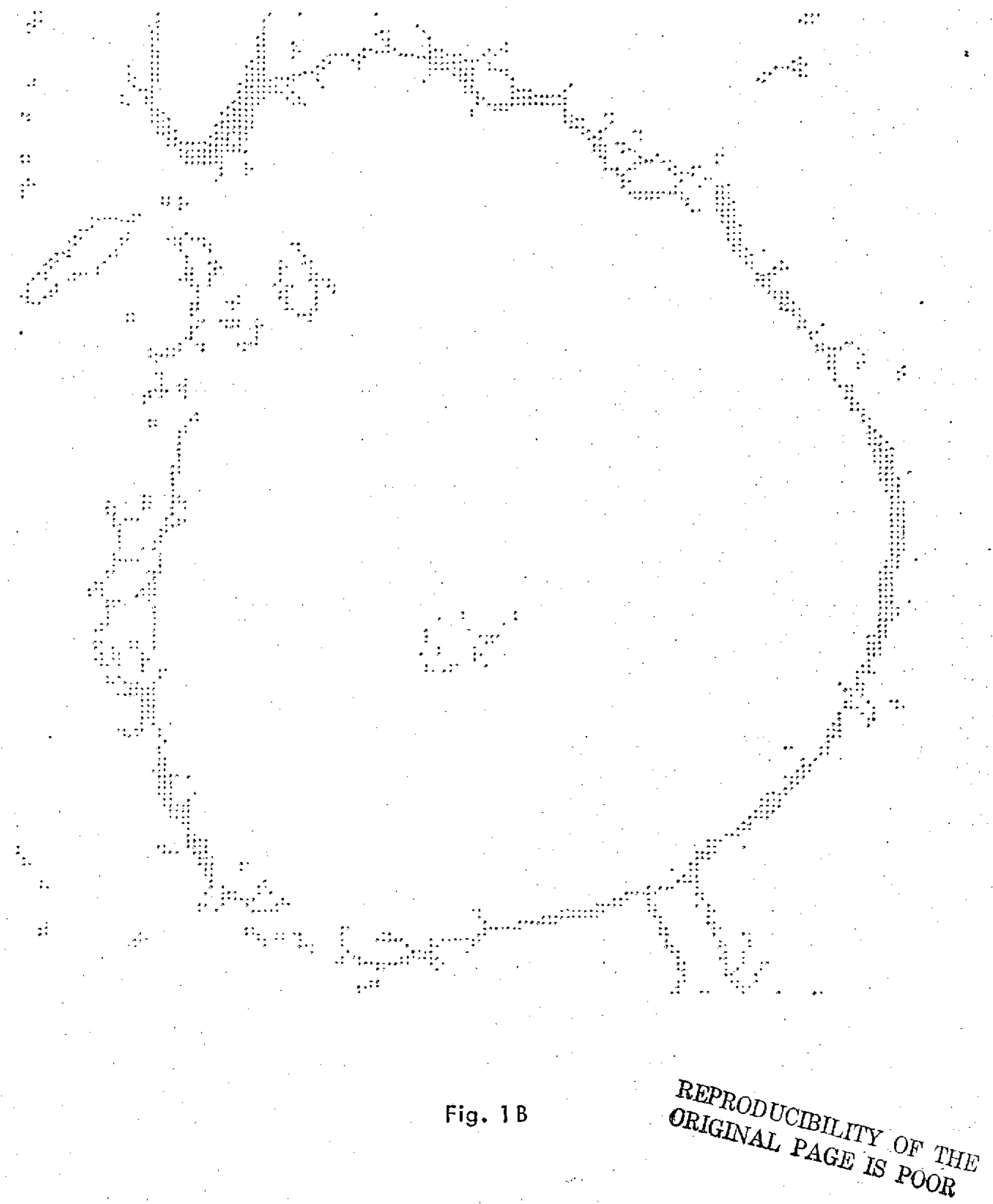




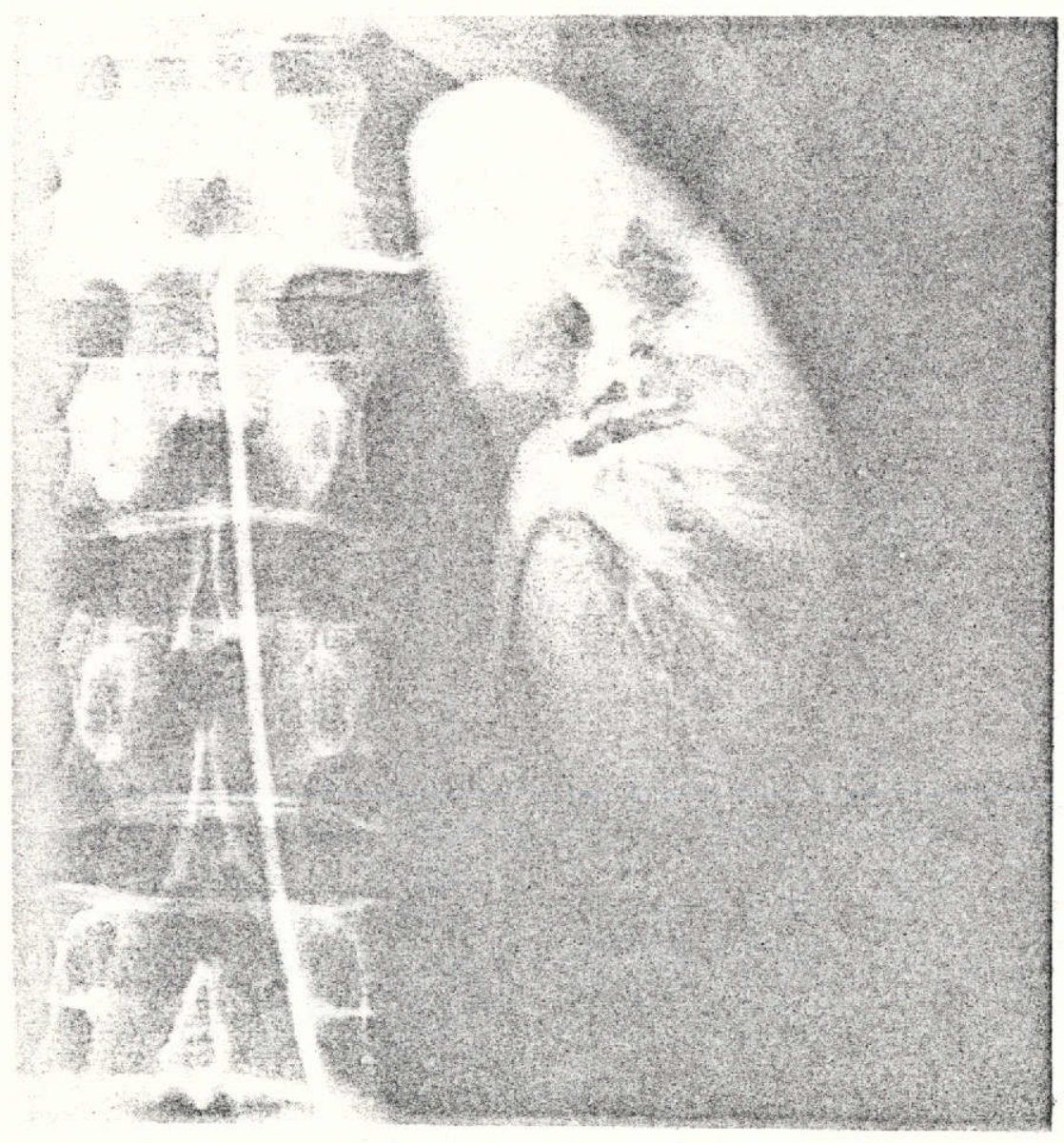

Fig. 2A

$$
\begin{aligned}
& \text { REPRODUCIBILITY OF THE } \\
& \text { ORIGLNAL PAGE IS POOR }
\end{aligned}
$$




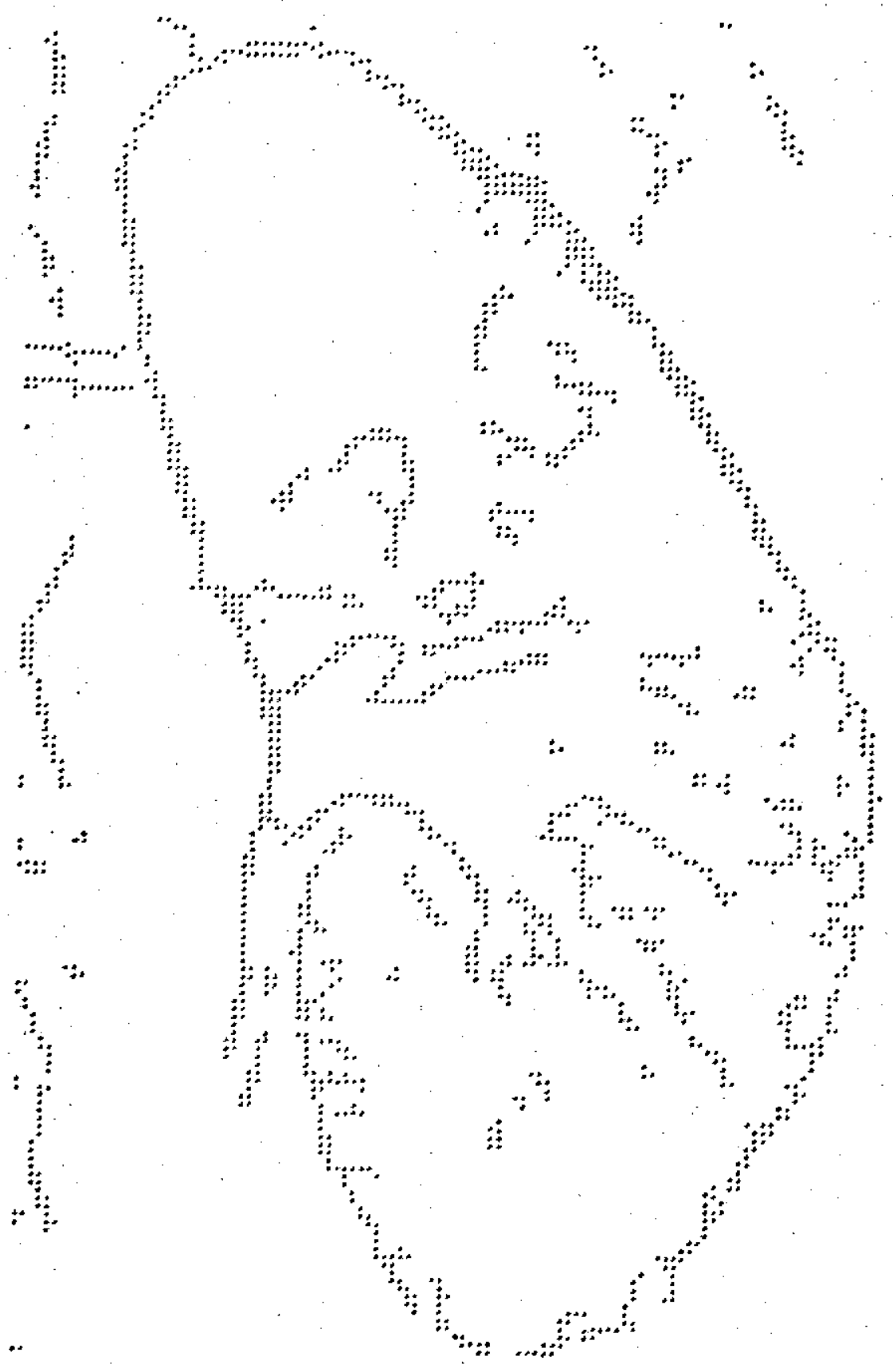

Fig. 2B

$$
\begin{aligned}
& \text { REPRODUCIBILITY OF THE } \\
& \text { ORIGLNAL PAGE IS POOR }
\end{aligned}
$$

